\title{
Viscous dark matter
}

\author{
Diego Blas, ${ }^{a}$ Stefan Floerchinger, ${ }^{a}$ Mathias Garny, ${ }^{a}$ \\ Nikolaos Tetradis, ${ }^{b}$ and Urs Achim Wiedemann ${ }^{a}$ \\ ${ }^{a}$ Physics Department, Theory Unit, CERN, CH-1211 Genève 23, Switzerland \\ ${ }^{b}$ Department of Physics, University of Athens, Zographou 157 84, Greece
}

\begin{abstract}
We discuss two aspects of the scenario in which dark matter possesses (fundamental or effective) viscosity: a) Large shear viscosity can induce the acceleration of the average expansion through the backreaction of fluctuations. b) Treating dark matter at large scales as an effectively viscous fluid provides an improved framework for the calculation of the matter power spectrum. We use intuition from the Wilsonian renormalization group in order to make this framework concrete, and review results that demonstrate that it improves the convergence of cosmological perturbation theory.
\end{abstract}

Keywords: Dark matter; viscosity; cosmological acceleration; power spectrum.

\section{Introduction}

The standard cosmological paradigm (the $\Lambda$ CDM model) assumes the presence of an energy component of the Universe termed dark matter, which, over sufficiently large scales, can be described as an ideal pressureless fluid. However, the exact nature of dark matter is still unknown, which raises the question of whether there is room or scope in going beyond this simplest assumption. The first-order formalism of relativistic fluid dynamics allows for bulk and shear viscosity. Endowing dark matter with such properties leads to modifications in the evolution of structure in the Universe, with observable consequences. We focus here on shear viscosity and neglect bulk viscosity. The role of the latter in cosmology has been studied extensively. ${ }^{1-4}$ Two aspects of shear viscous dynamics were explored recently in Refs. 5 and 6 , corresponding to viscosities of two qualitatively different origins:

- Fundamental (microscopic) viscosity: This viscosity is due to the diffusive transport of momentum, generated by the fundamental interactions between the particles that constitute the fluid.

- Effective viscosity: If one limits the fluid description to wavenumbers $k$ below a scale $k_{m}$, effective viscosities must be introduced in order to parametrize how momentum carried by the long-wavelength modes dissipates to shorter wavelengths $\left(k>k_{m}\right)$ that are not evolved explicitly. Here, the underlying mechanism is the interaction between long- and shortwavelength modes. An example is the description of approximately laminar flow on large scales in the presence of short-scale eddies (eddy viscosity).

We neglect the effect of baryons and discuss the evolution of the Universe in the framework of cosmological perturbation theory. We model dark matter as a relativistic viscous fluid with energy momentum tensor of the form

$$
T^{\mu \nu}=\rho u^{\mu} u^{\nu}+p \Delta^{\mu \nu}-2 \eta\left(\frac{1}{2}\left(\Delta^{\mu \rho} \nabla_{\rho} u^{\nu}+\Delta^{\nu \rho} \nabla_{\rho} u^{\mu}\right)-\frac{1}{3} \Delta^{\mu \nu}\left(\nabla_{\rho} u^{\rho}\right)\right) .
$$


Here $\rho$ is the energy density and $p$ the pressure in the fluid rest frame, $\eta$ is the shear viscosity, $\nabla_{\mu}$ denotes a covariant derivative, and $\Delta^{\mu \nu}=g^{\mu \nu}+u^{\mu} u^{\nu}$ projects to the subspace orthogonal to the fluid velocity. The velocity field $u^{\mu}$ can be expressed through the coordinate velocity $v^{i}=d x^{i} / d \tau$. The dynamics is governed by the Einstein equations and the conservation of the energy-momentum tensor.

\section{Backreaction Effect from Fundamental Shear Viscosity}

In cosmological perturbation theory all fields are split into a homogeneous and isotropic background and perturbations around it, e.g. $\rho(\tau, \mathbf{x})=\rho_{0}(\tau)+\delta \rho(\tau, \mathbf{x})$. Einstein's equations are non-linear, and, beyond first order, their spatial average differs from the equations for the spatially averaged fields. This effect is characterized as backreaction of the fluctuations on the background. If we assume that dark matter is pressureless, the averaged continuity equation takes the form ${ }^{5}$

$$
\frac{1}{a} \dot{\rho}_{0}+3 H \rho_{0}=D \equiv \frac{1}{a^{2}} \eta\left\langle\left[\partial_{i} v_{j} \partial_{i} v_{j}+\partial_{i} v_{j} \partial_{j} v_{i}-\frac{2}{3} \partial_{i} v_{i} \partial_{j} v_{j}\right]\right\rangle .
$$

We can combine this equation with the averaged trace of the Einstein equations

$$
\frac{\ddot{a}}{a^{3}}=\frac{1}{a} \dot{H}+2 H^{2}=\frac{4 \pi G_{\mathrm{N}}}{3} \rho_{0} .
$$

For the deceleration parameter $q=-1-\dot{H} /\left(a H^{2}\right)$ we find

$$
-\frac{d q}{d \ln a}+2(q-1)\left(q-\frac{1}{2}\right)=\frac{4 \pi G_{N} D}{3 H^{3}} .
$$

For $D=0$, this equation has an attractive fixed point at the standard value $q=1 / 2$. However, if the rhs is positive, the fixed point is shifted towards negative values of $q$. More specifically, the fixed point is accelerating, i.e. $q<0$, for $4 \pi G_{\mathrm{N}} D /\left(3 H^{3}\right)>1$. If the fundamental properties of dark matter result in substantial shear viscosity, the backreaction on the average expansion may cause it to accelerate.

In order to examine if the condition $4 \pi G_{\mathrm{N}} D /\left(3 H^{3}\right)>1$ can be satisfied, we need to estimate the various terms in $D$. We can assume that typical gradients of the fluid velocity are of the same order as the Hubble rate $H$, so that $\eta\left\langle\partial_{i} v_{j} \partial_{i} v_{j}+\partial_{i} v_{j} \partial_{j} v_{i}-\right.$ $\left.\frac{2}{3} \partial_{i} v_{i} \partial_{j} v_{j}\right\rangle / a^{2} \sim \eta H^{2}$. This corresponds to realistic peculiar velocities of the order of $100 \mathrm{~km} / \mathrm{s}$ over distances of order $1 \mathrm{Mpc}$. A similar estimate can be obtained from the power spectrum of the velocity field within cosmological perturbation theory. The viscosity coefficient $\eta$ depends on the unknown properties of the dark sector. A large shear viscosity arises for systems containing very weakly interacting relativistic particles of long mean free paths ${ }^{7}$ (possibly forming an additional component to standard cold dark matter). In this case, relativistic kinetic theory suggests that ${ }^{8}$ $\eta \sim \rho \tau$, where $\rho$ is the energy density carried by the weakly interacting particles and $\tau$ is their mean free time. Accelerating expansion would result if $4 \pi G_{\mathrm{N}} D /\left(3 H^{3}\right) \sim$ $\rho \tau H /\left(2 \rho_{c}\right)$ is of order unity, where $\rho_{c}$ is the critical density, defined through $H^{2}=$ $8 \pi G_{\mathrm{N}} \rho_{c} / 3$. On the other hand, for a fluid description to be applicable, the mean free time must be smaller than the expansion rate, $\tau H<1$. Thus, for shear viscosity 
to have a significant effect, $\rho$ must be of the same order as $\rho_{c}$. The construction of detailed models with a dark-matter component with these properties is a very interesting research direction. Some speculative suggestions in this direction can be found in Ref. 5 .

\section{Effective Viscosity and Large-Scale Structure}

The description of large-scale fluctuations can profit from the notion of an effective viscosity and pressure even if dark matter behaves as an ideal pressureless fluid at the fundamental level. Effective viscosity and pressure allow the parametrization of the interaction of large-scale fluctuations $\left(k<k_{m}\right)$ with those short-scale ones $\left(k>k_{m}\right)$ that are not followed in a fluid dynamical description limited to $k<k_{m}$. In principle, one could dispense with this effective viscosity and pressure by following the dynamics on all scales, but in practice absorbing effects from small scales in a few effective parameters is much more efficient.

The fluid dynamic formulation that we introduce relies on the notion of coarse graining. As discussed in Ref. 6, this formulation shares conceptual points with the effective field theory approach of Ref. 9. However, there are crucial differences: our calculation is self-contained and does not rely on external information from $N$-body simulations; we fully include the viscosity and pressure terms in the propagator, instead of treating them perturbatively, thus obtaining a better UV behavior; we keep a finite, and rather low, UV cutoff in the effective theory, thus reducing the sensitiviy of our results on the complicated short-scale dynamics.

The large-scale description in terms of an imperfect fluid was employed in Ref. 6 in order to obtain an accurate estimate of the matter power spectrum at the scales of baryon acoustic oscillations (BAO). In addition to the motivation for this framework given in Ref. 6, we provide here a somewhat different, intuitive motivation, based on the Wilsonian Renormalization Group. We also summarize the main results and refer the reader to Ref. 6 for a thorough discussion of the technical aspects of the calculation of the spectrum.

\subsection{Intuition from the Wilsonian Renormalization Group}

We draw inspiration from the Wilsonian approach to the renormalization group, in the formulation of the effective average action (for a review see Ref. 10). Let us consider a theory of a real scalar field $\chi$, in $d$ dimensions, with a $Z_{2}$-symmetric action $S[\chi]$. We add to the kinetic term a regulating piece

$$
\Delta S=\frac{1}{2} \int d^{d} q \hat{R}_{k}(q) \chi^{*}(q) \chi(q) .
$$

The function $\hat{R}_{k}$ is employed in order to prevent the propagation of modes with momenta $q^{2} \lesssim k^{2}$. For such modes, the effective propagator $P(q)=\left(q^{2}+\hat{R}_{k}(q)\right)^{-1}$ is suppressed by arranging for $\hat{R}_{k}(q)$ to be large. On the other hand, $\hat{R}_{k}(q)$ vanishes for $q^{2} \gtrsim k^{2}$. The form of $\hat{R}_{k}$ is not unique, and many alternative choices are possible. 
In particular, the effective cutoff $k$ can be made sharp through an appropriate choice of $\hat{R}_{k}(q)$. Through the standard Legendre transformation one obtains the generating functional for the 1PI Green functions $\tilde{\Gamma}_{k}[\phi]$, where $\phi$ is the expectation value of the field $\chi$ in the presence of sources. The use of the modified propagator for the calculation of $\tilde{\Gamma}_{k}$ results in the effective integration of only the fluctuations with $q^{2} \gtrsim k^{2}$. Finally, the cutoff-dependent effective average action is obtained by subtracting the regulating term

$$
\Gamma_{k}[\phi]=\tilde{\Gamma}_{k}[\phi]-\frac{1}{2} \int d^{d} q \hat{R}_{k}(q) \phi^{*}(q) \phi(q) .
$$

We identify the tree-level action $S$ with $\Gamma_{k}$ at some fundamental scale $k=\Lambda$ (no effective integration of modes takes place). In the opposite limit $k \rightarrow 0$ one obtains the standard effective action. An exact flow equation describes the response of $\Gamma_{k}$ to variations of the infrared cutoff $(t=\ln k)^{11}$

$$
\frac{\partial \Gamma_{k}[\phi]}{\partial t}=\frac{1}{2} \operatorname{Tr}\left[\left(\Gamma_{k}^{(2)}[\phi]+\hat{R}_{k}\right)^{-1} \frac{\partial \hat{R}_{k}}{\partial \ln k}\right] .
$$

Here $\Gamma_{k}^{(2)}$ is the second functional derivative of $\Gamma_{k}$ with respect to $\phi$.

In order to extract practical information from eq. (7), one must introduce some truncation scheme, such as a derivative expansion of $\Gamma_{k}[\phi]$. For the simplest truncation, which keeps a general potential $U_{k}(\phi)$ and a standard kinetic term, one obtains

$$
\frac{\partial U_{k}(\phi)}{\partial \ln k}=\frac{1}{2} \int \frac{d^{d} q}{(2 \pi)^{d}} \frac{\partial \hat{R}_{k}(q)}{\partial \ln k} \frac{1}{q^{2}+\hat{R}_{k}(q)+U_{k}^{\prime \prime}(\phi)} .
$$

Contact with standard perturbation theory can be achieved through an iterative solution of eq. (8). At the level of the first iteration, the rhs is evaluated in terms of the tree-level potential $U_{\Lambda}$. Using a sharp cutoff, one obtains

$$
U_{k}(\phi)=U_{\Lambda}(\phi)+\frac{1}{2} \int_{k}^{\Lambda} \frac{d^{d} q}{(2 \pi)^{d}} \ln \left(q^{2}+U_{\Lambda}^{\prime \prime}(\phi)\right) .
$$

The effective average action $\Gamma_{k}[\phi]$ includes all the terms consistent with the symmetries of the system. In particular, the coarse-grained potential $U_{k}(\phi)$ includes couplings that may not be present in the tree-level potential $U_{\Lambda}(\phi)$, but result from the integration of modes with $k^{2} \lesssim q^{2} \lesssim \Lambda^{2}$. Repeating this procedure leads to the determination of the effective potential $U(\phi) \equiv U_{0}(\phi)$ in terms of $U_{k}(\phi)$ through a relation similar to $(9)$, but with the limits of the momentum integration becoming 0 and $k$. The coarse-graining scale $k$ acts as an UV cutoff in this integration, because the effect of the modes with higher momenta has been incorporated in the effective couplings. Following the above procedure for an infinite number of infinitesimal $k$-intervals is equivalent to the solution of eq. (8).

We apply the spirit of the Wilsonian formulation that we described above to the description of large-scale cosmological perturbations. Our analysis is based on the 
effective theory that results from the coarse graining of the short-scale fluctuations. The quantities of interest are the spectra of perturbations. The main conceptual points of our scheme are:

- The large-scale description may include additional interactions, not necessarily present in the fundamental description. The commonly employed derivative expansion of the effective average action arranges the various terms according to the number of field derivatives. The action is then truncated, keeping the lowest-order terms. If dark matter is modelled as a fluid, a similar procedure would keep the terms with the smallest number of derivatives in the energy-momentum tensor. At the level of the first-order formalism, one must include viscosity terms.

- The large-scale theory must come with an UV cutoff, set by the coarsegraining scale. Our choice for this scale will be in the region $0.4-1 \mathrm{~h} / \mathrm{Mpc}$.

The analogy with field theory is not exact for various reasons:

- The evolution of large-scale structure has an explicit time dependence, while the coarse graining concerns only the spatial part. (For this reason, the relevant momentum integrations have $d=3$.)

- The cosmological fluctuations develop from stochastic fluctuations in the initial state, with a characteristic primordial spectrum. The usual formulation of the Wilsonian renormalization group for quantum or thermal fluctuations in Euclidean space is not directly applicable.

- The "tree-level" description at very short scales and late times is not known for the problem of cosmological perturbations. A fluid description can be derived from kinetic theory under certain assumptions, such as the singlestream approximation and the presence of a smoothing scale. However, it is expected to break down at the typical length scales of virialized structures.

- Even before the breakdown of the fluid description, perturbation theory stops converging. Higher loops display larger sensitivity to the deep UV.

Despite these limitations, an efficient framework for the treatment of cosmological perturbations can be constructed in analogy to the Wilsonian approach.

\subsection{The matter power spectrum within the coarse-grained theory}

Based on our previous discussion, we develop a viscous-fluid desciption for cosmological perturbations with wavenumbers $k<k_{m}$. Here $k_{m}$ is a cutoff scale chosen such that strongly non-linear perturbations have wavenumber larger than $k_{m}$. Perturbations with $k>k_{m}$ are not evolved explicitly, but their effect on the long wavelength modes is parametrized in terms of effective viscosity and pressure (or sound velocity). In analogy to eq. (9), these effective couplings are determined by a one-loop expression in the perfect-fluid, pressureless theory, which involves a momentum integral over the range $k>k_{m}$. We have found that the most efficient 
$P_{\delta \delta}(\mathrm{k}, \mathrm{Z}=0)$

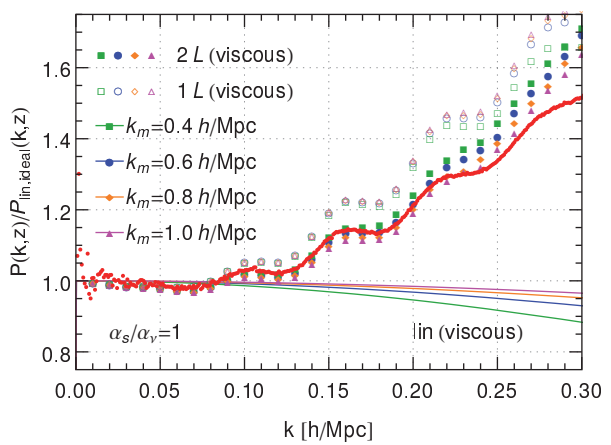

$P_{\delta \delta}(\mathrm{k}, \mathrm{z}=0.375)$

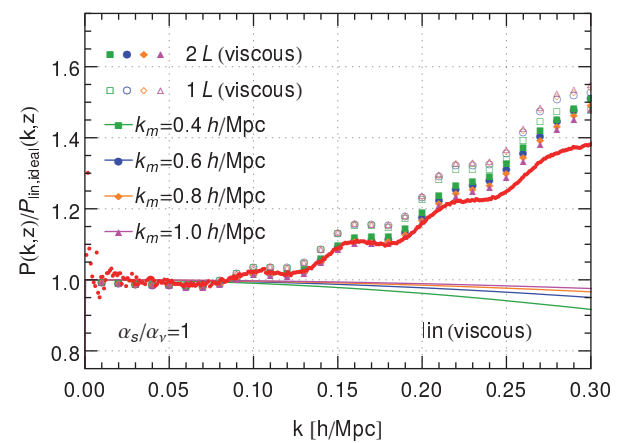

Fig. 1. Dependence of the power spectrum on the scale $k_{m}$, for $z=0$ and $z=0.375$. The coloured lines show the linear spectra, the open and filled symbols the one- and two-loop spectra, for various values of $k_{m}$. The red points, merging into thick red lines, show the results of the Horizon $N$-body simulation. ${ }^{13}$

way to determine the couplings is through the effective propagator

$$
G_{a b}\left(\mathbf{k}, \tilde{\eta}, \tilde{\eta}^{\prime}\right) \delta^{(3)}\left(\mathbf{k}-\mathbf{k}^{\prime}\right)=\left\langle\frac{\delta \phi_{a}(\mathbf{k}, \tilde{\eta})}{\delta \phi_{b}\left(\mathbf{k}^{\prime}, \tilde{\eta}^{\prime}\right)}\right\rangle,
$$

which defines the response of the field at late times $\tilde{\eta}$ to a perturbation at earlier times $\tilde{\eta}^{\prime}$. We require that the tree-level propagator of the viscous theory is identical to the propagator calculated from standard perturbation theory beyond linear order in the perfect-fluid theory, when only the fluctuations with $k>k_{m}$ are taken into account. The matching can be achieved with an accuracy at the percent level. The relevant quantity appearing in the expressions for the effective viscosity and sound velocity is 6

$$
\sigma_{d, \text { match }}^{2} \equiv \frac{4 \pi}{3} \int_{k_{m}}^{\infty} d q P^{L}(q),
$$

with $P_{L}$ the linear density-density spectrum of the perfect-fluid theory. This expression allows us to fix the redshift dependence of the effective couplings, which is determined through the linear growth factor. The range of integration includes the deep UV region in which perturbation theory is expected to fail. However, the form of the linear spectrum, which scales $\sim k^{-3}$ (up to logarithmic corrections) for large $k$ implies that the integral is dominated by the region near $k_{m}$, where perturbation theory is still applicable. There is independent evidence from $N$-body simulations that the effect of UV modes on the IR ones through mode-mode coupling is weak. ${ }^{12}$ It is apparent that $k_{m}$ cannot be taken arbitrarily large (we take it in the region $0.4-1 h / \mathrm{Mpc}$ ), while physical quantities should be independent of its value.

In fig. 1 we show the matter power spectrum at two redshifts, computed for a viscous fluid in the effective theory with an UV cutoff $k_{m}$. All curves are normalized to the linear spectrum obtained for an ideal, pressureless fluid. The solid lines 
$P_{\delta \delta}(\mathrm{k}, \mathrm{z}=0) / P_{N-\text { body }}$, viscous theory

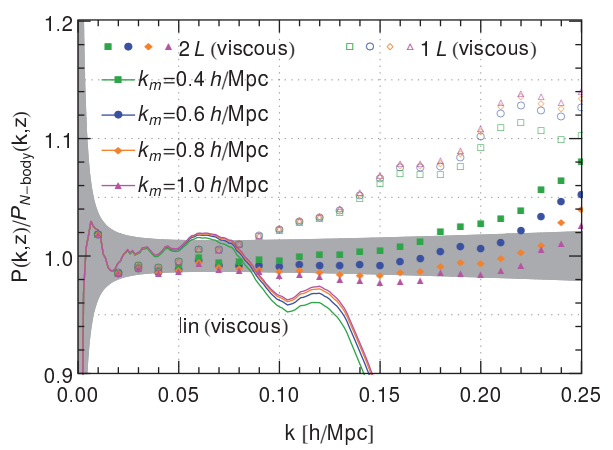

$P_{\delta \delta}(\mathrm{k}, \mathrm{Z}=0) / P_{N-\text { body }}, \mathrm{SPT}$ with cutoff

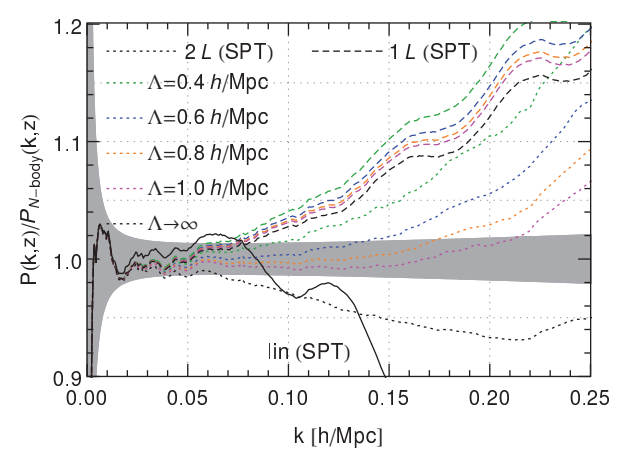

Fig. 2. Comparison of results within the viscous theory (left) and in standard perturbation theory (right). The spectra are normalized with respect to the $N$-body result ${ }^{13}$ at $z=0$. The grey band indicates an estimate for the error of the $N$-body simulation. The left plot shows one- and two-loop results within the viscous theory for various values of $k_{m}$. The right plot shows one- and two-loop results in standard perturbation theory, computed with a UV cutoff $\Lambda$ equal to $k_{m}$ (coloured lines), as well as when the cutoff is removed (black lines).

show the linear spectrum in the viscous theory. The open (filled) circles show the one-loop (two-loop) result, taking the time- and scale-dependent viscosity and pressure terms into account, and including modes with $k<k_{m}$ in the loop integrals. For comparison, the red points show corresponding results from a large-scale $N$ body simulation. ${ }^{13}$ The two-loop results agree very well with the $N$-body data for $k \lesssim 0.2 h / \mathrm{Mpc}$ at low redshift, and the agreement improves at higher redshift. In the same figure we can also see the dependence on $k_{m}$ in the range $0.4-1 h / \mathrm{Mpc}$. The variation of the two-loop results is small within the range for which the results agree well with $N$-body data.

In fig. 2 we compare our results (left plot) in the viscous theory with standard perturbation theory results (right plot). The two-loop results within standard perturbation theory depend strongly on the cutoff $\Lambda$. In contrast, the two-loop results within the viscous theory have a very mild dependence on the variation of the matching scale $k_{m}$ over the range $0.4-1 h / \mathrm{Mpc}$. They display a better agreement with $N$-body data compared to standard perturbation theory. The plost also demonstrate that the convergence of the loop expansion within the viscous theory is improved compared to standard perturbation theory.

\section{Conclusions}

We examined the physical consequences of treating dark matter as an imperfect fluid. Within the first-order formalism we explored novel effects associated with shear viscosity and nonzero sound velocity, focusing on the role of fluctuations. We argued that, if viscosity is sufficiently large, it can induce the dissipation of energy from the fluctuations to the homogeneous background at a rate sufficiently fast for 
the cosmological expansion to become accelerating. This scenario requires that dark matter possesses large fundamental (microscopic) viscosity. This possibility deserves further investigation, since the nature of dark matter still remains unknown.

Apart from the possible presence of fundamental viscosity, the large-scale description of dark matter requires the introduction of effective viscosity and pressure. In order to reinforce the arguments of Ref. 6, we summarized the lessons provided by the Wilsonian renormalization group for the calculation of these effective couplings. We discussed how one can isolate the effect on the low- $k$ modes arising from modes near and slightly above $1 \mathrm{~h} / \mathrm{Mpc}$, and translate it into effective low-energy parameters (viscosity and sound velocity). There is strong evidence that these give the dominant effect, while the influence of the deep UV is negligible. One can then use perturbation theory within the low- $k$ theory, cutting off all integrations with an upper limit equal to $k_{m}$. We found quick convergence of the perturbative series. On the other hand, standard perturbation theory becomes increasingly sensitive to the deep UV at higher loops, a feature that prohibits its convergence.

It is conceivable that the complete picture of the dark-matter sector may require the inclusion of both effective viscosity at large scales and fundamental viscosity for the description of the complicated dynamics at the shorter galaxy scales.

\section{References}

1. R. Maartens, Class. Quant. Grav. 12 (1995) 1455.

2. W. Zimdahl, Phys. Rev. D 53 (1996) 5483 [astro-ph/9601189].

3. J. S. Gagnon and J. Lesgourgues, JCAP 1109 (2011) 026 [arXiv:1107.1503 [astroph.CO]].

4. H. Velten, D. J. Schwarz, J. C. Fabris and W. Zimdahl, Phys. Rev. D 88 (2013) 10, 103522 [arXiv:1307.6536 [astro-ph.CO]].

5. S. Floerchinger, N. Tetradis and U. A. Wiedemann, Phys. Rev. Lett. 114 (2015) 9, 091301 [arXiv:1411.3280 [gr-qc]].

6. D. Blas, S. Floerchinger, M. Garny, N. Tetradis and U. A. Wiedemann, JCAP 1511 (2015) 11, 049 [arXiv:1507.06665 [astro-ph.CO]].

7. S. Weinberg, Astrophys. J. 168 (1971) 175.

8. S. Weinberg, Cosmology, Oxford University Press, Oxford (2008).

9. J. J. M. Carrasco, M. P. Hertzberg and L. Senatore, JHEP 1209 (2012) 082 [arXiv:1206.2926 [astro-ph.CO]].

10. J. Berges, N. Tetradis and C. Wetterich, Phys. Rept. 363 (2002) 223 [hep-ph/0005122].

11. C. Wetterich, Phys. Lett. B 301 (1993) 90.

12. T. Nishimichi, F. Bernardeau and A. Taruya, arXiv:1411.2970 [astro-ph.CO].

13. J. Kim, C. Park, G. Rossi, S. M. Lee and J. R. Gott III, J. Korean Astron. Soc. 44 (2011) 217 [arXiv:1112.1754 [astro-ph.CO]]. 